\title{
Correction to: Postoperative recurrence from tract seeding after use of EUS-FNA for preoperative diagnosis of cancer in pancreatic tail
}

\author{
Utae Sakamoto ${ }^{1} \cdot$ Nobuhiko Fukuba $^{1,3}$ (1) Shunji Ishihara ${ }^{1} \cdot$ Shohei Sumi ${ }^{1} \cdot$ Mayumi Okada $^{1} \cdot$ Hiroki Sonoyama $^{1}$. \\ Naoki Ohshima ${ }^{1} \cdot$ Ichiro Moriyama $^{2} \cdot$ Kousaku Kawashima ${ }^{1} \cdot$ Yoshikazu Kinoshita ${ }^{1}$
}

Published online: 5 March 2018

(c) Japanese Society of Gastroenterology 2018

\section{Correction to: Clinical Journal of Gastroenterology https://doi.org/10.1007/s12328-018-0822-z}

The original article has been corrected.

The correct name of the corresponding author should be "Nobuhiko Fukuba", and not "Nobuhiko Fukuban" as given in the original publication of the article.

The original article can be found online at https://doi.org/10.1007/ s12328-018-0822-z.

Nobuhiko Fukuba fnat98@yahoo.co.jp

1 Department of Internal Medicine II, Shimane University Faculty of Medicine, Izumo, Japan

2 Division of Cancer Center, Shimane University Hospital, Izumo, Japan

3 Department of Internal Medicine, Izumo City General Medical Center, 613, Nadabun-cho, Izumo, Shimane, Japan 